\title{
Erratum
}

\section{Computer Generation of Chemical Structures from Known Fragments}

\author{
Marko Razinger, Jure Zupan, and Marjana Novič
}

Mikrochim. Acta [Wien] 1986 II, 411-421

In this paper Fig. 3 on p. 418 was incorrect. The correct Fig. 3 should be:

Type in the numbers of selected fragments: 4, 5, 33, 33, 30, 34
1. $-\mathrm{H}$
30. $-\mathrm{OH}$
2. $-\mathrm{CH}_{3}$
31. $-\mathrm{COOH}$
3. $-\mathrm{CH}_{2}-$
32. $=\mathrm{O}$
4. $=\mathrm{CH}-$
33. $=\mathrm{CO}$
5. $=\mathrm{CCH}_{3}-$
34. $-\mathrm{NH}_{2}$
6. $=\mathrm{C}=\mathrm{C}=$
35. $-\mathrm{NH}-$
7. $-\mathrm{C} \equiv \mathrm{C}-$
36. $-\mathrm{N}=$
8. $\mathrm{CH}_{3}-\mathrm{S}-$
37. $-\mathrm{F}$
9. $\equiv \mathrm{C}-\mathrm{CH}_{2}-\mathrm{S}-$
38. $-\mathrm{Cl}$
10. $(=\mathrm{C}-)_{2} \mathrm{CH}-\mathrm{S}-$
39. $-\mathrm{Br}$
11. $(\equiv \mathrm{C}-)_{3} \mathrm{C}-\mathrm{S}-$
40. - I
12. $\mathrm{CH}_{3}-\mathrm{O}-$
41. $-\mathrm{SH}$
13. $\equiv \mathrm{C}-\mathrm{CH}_{2}-\mathrm{O}-$
42. $-\mathrm{Ph}$
14. $(\equiv \mathrm{C}-)_{2} \mathrm{CH}-\mathrm{O}-$
43. $-\mathrm{CH}_{2}-\mathrm{Ph}$
15. $(\equiv \mathrm{C}-)_{3} \mathrm{C}-\mathrm{O}-$
44. $-\mathrm{O}-\mathrm{Ph}$
16. $\equiv \mathrm{C}-\mathrm{CH}_{2}-\mathrm{NO}_{2}$
45. $-\mathrm{S}-$

Fig. 3. A part of the menu, used for the definition of the chemical nature of fragments. For two different trivalent, two identical bivalent and two different monovalent fragments (corresponding to the input sequence in Fig. 1), entries 4 and 5 , two 33,30 and 34 were chosen, respectively 